\title{
Electromagnetic Wave Propagation in Circular Tunnels
}

\author{
Osama M. Abo-Seida \\ Mathematics Department, Faculty of Science, Kafr El-Sheikh University, \\ Egypt
}

\section{Introduction}

The problem of guided wave propagation in a wave guide with imperfectly reflecting boundaries arises in several applications, such as propagation in mine tunnels and in screened surface wave guides. In recent years, many studies have been carried out on the propagation characteristic of radio waves in tunnels in UHF band [1-2]. From the theoretical point of view, tunnels can be regarded as hollow waveguides surrounded by a lossy dielectric medium, such as concrete, ground, and so on. The problem of radio communication in tunnels has found solutions using leaky transmission lines as supports for propagation of transverse electromagnetic modes [3-4]. These modes are characterized by the fact that there is no cutoff frequency, and by an attenuation which increases with increasing frequency. However, when the frequency is high enough, natural propagation modes, which are transverse electric or transverse magnetic, can appear and interfere with the transmission line supported transverse electromagnetic modes [5].

Natural propagation can be helpful to solve some specific problems, such as radio communications in mines with rooms and pillars which cannot be solved easily with the help of transmission lines. Natural propagation modes are also useful for short range communications, for example, in some road tunnels. For distances shorter than $200 \mathrm{~m}$, these modes can be more suitable the transmission line supported modes [6].

In order to maximize the performance attainable feeding in the interior of tunnels with antenna systems, it is important to estimate the value and the attenuation of the electromagnetic strength inside the tunnel. Investigations concerning radiowave propagation in railways tunnels have been performed [7] and the results confirm the existence of a waveguide effect strongly related to the antenna positions. Recently, AboSeida et al. [8] and Abo-Seida [9] studied the electromagnetic field due to vertical magnetic dipole buried in stratified media. Also, Abo-Seida [10] computed the attenuation below and above the cutoff frequency in a rectangular tunnel.

It might be thought that waveguides with circular cross sections would be preferred to guides with rectangular cross sections, just as circular pipes are commonly used for carrying water and fluids in preference to rectangular pipes. However, circular waveguides have the disadvantage that there is only a very narrow range between the cutoff wavelength of the dominant mode and the cutoff wavelength of the next higher mode. As with rectangular guides, the modes may be classified as transverse electric ( TE ) or transverse magnetic ( TM ), according to whether it is the electric or magnetic lines of force that lie in planes perpendicular 
to the axis of the guide. The different modes are designated by a double subscript system analogous to that for rectangular guides.

\section{Geometrical structure and basic equations}

The concrete tunnel is practically of finite thickness. At low frequencies, below cutoff frequencies, the skin depth may exceed this thickness and thus the field penetrates to the outside. However, our aim is to check the possibilities of radio communication in tunnels and to replace the tunnel by a circular waveguide. It is possible to make this replacement in practical problems involving conducting walls.

A uniform waveguide of circular cross section is most conveniently described by polar coordinate system $(r, \phi, z)$, and divide the possible solutions for circular guides into transverse magnetic and transverse electric waves. For the TM waves $H_{z}$ is identically zero and the wave equation for $E_{z}$ is used. Also, TE waves $E_{z}$ is identically zero and $H_{z}$ is used. The modes are further labeled by a two-dimensional order number $(m, n)$.

For a circular waveguide with a radius $r$ the critical frequencies are given by

$$
f_{c m n}=\left[\begin{array}{ll}
\frac{C Y_{m n}}{2 r}, & T M_{m n} \bmod e s \\
\frac{C Y_{m n}^{\prime}}{2 r}, & T E_{m n} \operatorname{modes}
\end{array}\right.
$$

where $m \geq 0, Y_{m n}$ and $Y_{m n}^{\prime}$ are the nth zero of Bessel function $J_{m}$ and of its derivative, respectively, and $C=3 \times 10^{8} \mathrm{~ms}^{-1}$ is the speed of light.

The cutoff wavelengths are given for a circular waveguide by [11]

$$
\lambda_{c m n}=\left[\begin{array}{lc}
\frac{2 \pi}{Y_{m n}} r, & T M_{m n} \bmod e s \\
\frac{2 \pi}{Y_{m n}^{\prime}} r, & T E_{m n} \bmod e s
\end{array}\right.
$$

where $m, n$ are equal to $1,2,3, \ldots \ldots .$. for the $T M_{m n}$ modes, and equal to $0,1,2,3, \ldots \ldots$. for the $T E_{m n}$ modes.

Below the lowest cutoff frequency, propagation is not possible. The attenuation $\alpha$ is independent of the electrical properties of the wall and have the form equation

$$
\begin{gathered}
\alpha=\frac{2 \pi}{\lambda_{c}} \sqrt{1-\left(\frac{\lambda_{c}}{\lambda}\right)^{2}}=\frac{2 \times 3.14 \times 8.69}{\lambda_{c}} \sqrt{1-\left(\frac{\lambda_{c}}{\lambda}\right)^{2}} \\
=\frac{54.6}{\lambda_{c}} \sqrt{1-\left(\frac{\lambda_{c}}{\lambda}\right)^{2}} \mathrm{~dB} / \text { unit length }
\end{gathered}
$$

where $\lambda_{c}$ is the longest cutoff wavelength of the waveguide and the value 8.69 is the decibels of attenuation per unit length.

Above its cutoff frequency, the attenuation $\alpha$ for the $T M_{m n}$ modes is given by [11] 


$$
\alpha=8.69 \frac{R}{\xi r} \frac{1}{\sqrt{1-\left(\frac{\lambda}{\lambda_{m n}}\right)^{2}}} \mathrm{~dB} / \mathrm{m}
$$

and for the $T E_{m n}$ modes, this attenuation $\alpha$ is given by

$$
\alpha=8.69 \frac{R}{\xi r}\left[\frac{m^{2}}{Y_{m n}^{\prime 2}-m^{2}}+\left(\frac{\lambda}{\lambda_{m n}}\right)^{2}\right] \frac{1}{\sqrt{1-\left(\frac{\lambda}{\lambda_{m n}}\right)^{2}}} \mathrm{~dB} / \mathrm{m}
$$

where ${ }_{1} \xi$ is the intrinsic impedance of the propagation medium and equal to $(\mu / \varepsilon)^{2}, R=10.88 \times 10^{-3} \sqrt{\left(10^{7} / \sigma\right)(1 / \lambda)}$ ohms, $\sigma$ is the conductivity of the guide walls in $\mathrm{mho} / \mathrm{m}, \mu$ is the permeability of the propagation medium in henry/m, $\varepsilon$ is the permittivity of the propagation medium in $\mathrm{farad} / \mathrm{m}$.

\section{Determination of the attenuation constant}

We consider the circular -waveguide model of the tunnel. In practice, the concrete tunnel wall is of finite thickness. This tunnel is considered to have a radius $r=4.08 \mathrm{~m}$. Its conductivity is taken as $10^{-1}$ and $10^{-2} \mathrm{mho} / \mathrm{m}$ as in [10].

The cutoff frequency is $20 \mathrm{MHz}$ corresponding to approximately a tunnel shaped as a circular cylinder, inside it a wave is propagating in the

$T E_{11}$ mode. Taking account of the conductivity of the walls, the attenuation constants of the $T E_{11}$ and $T E_{01}$ modes have been determined and the results plotted in Figs (1) and (2), respectively, for both values of $\sigma$ and for frequencies in the range 20-300 $\mathrm{MHz}$.

As in the case of $T E_{m n}$ modes, the attenuation of the electromagnetic waves is calculated for both the $T M_{11}$ and $T M_{01}$ modes. The obtained results are plotted in Figs (3) and (4), respectively, as a function of the frequency.

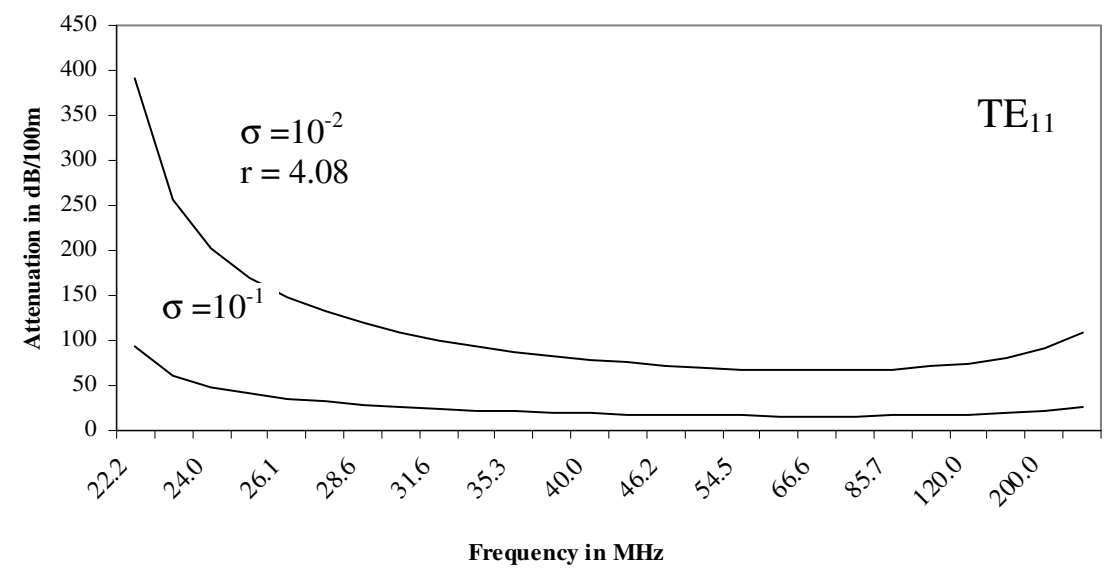

Fig. 1. Frequency and attenuation in a circular tunnel 


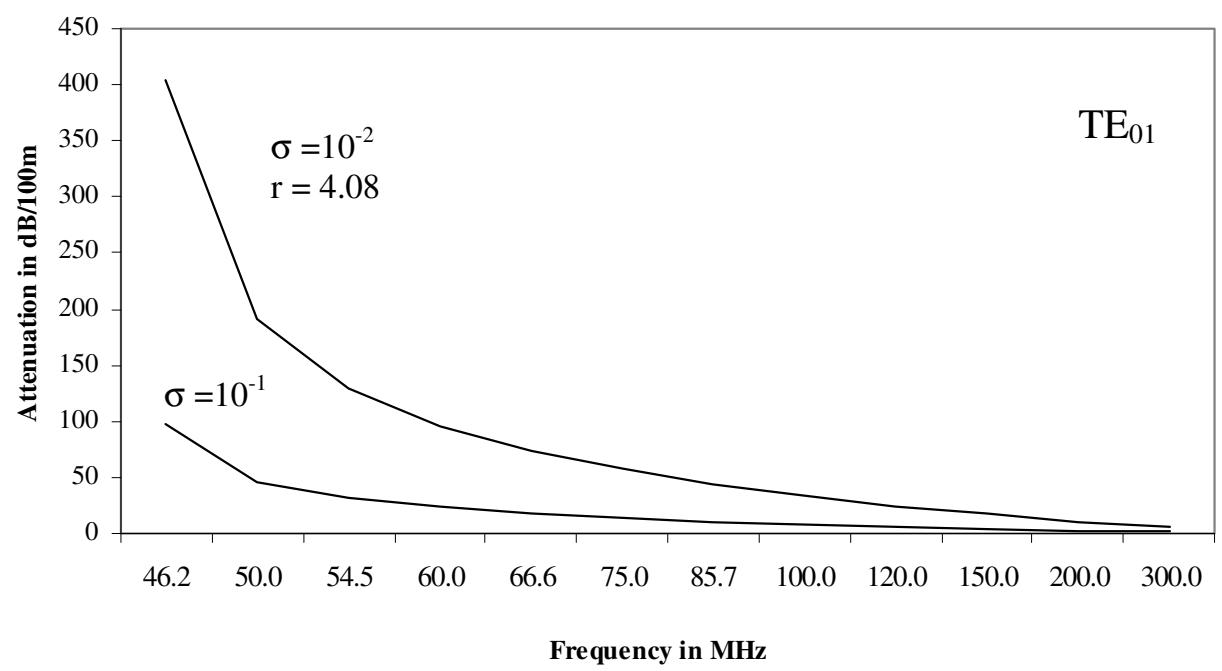

Fig. 2. Frequency and attenuation in a circular tunnel

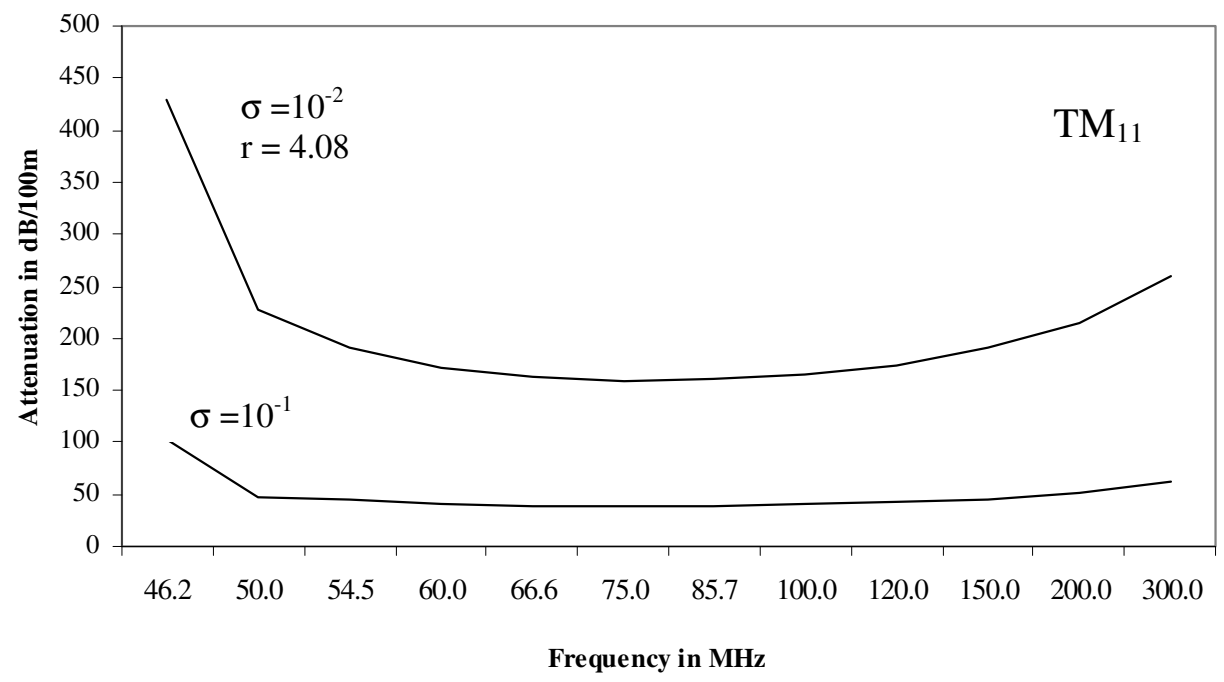

Fig. 3. Frequency and attenuation in a circular tunnel 


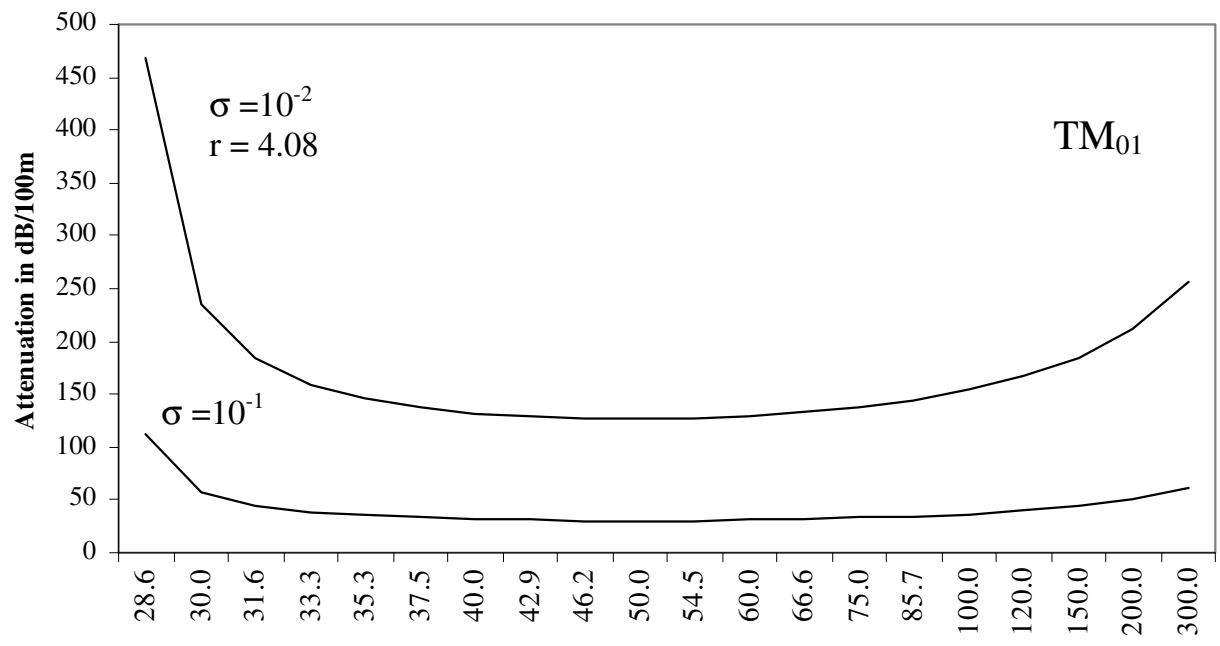

Frequency in MHz

Fig. 4. Frequency and attenuation in a circular tunnel

\section{Conclusion}

The propagation of electromagnetic waves in a circular tunnel have been performed and the results confirm the existence of a waveguide effect strongly related to the antenna positions. The cutoff frequency was calculated and three different types of frequency ranges were characterized. The numerical results presented here indicate that the different values of $\mathrm{m}$ and $n$ modes.

This work enable us to distinguish three different ranges of frequency, characterized by three different propagation mechanisms.

\section{References}

Emslie, A.; Lagace, R. \& Strong, P.(1975). Theory of the propagation of UHF radio waves in cool mine tunnels, IEEE Trans. Antennas Propagat. AP-23, pp. 192-205.

Delogne, P. (1982). Leaky feeders and subsurface radio communication, Peter Peregrinus, Stevegage, U.K.

Delogne,P. \& Safak, M. (1975). Electromagnetic Theory of the leaky, Coaxial Cable, The Radio and Electronic Engineer, vol. 45, pp. 233-240.

Deryck, L. (1975). Control of mode conversions on bifilar line in tunnels, The Radio and Electronic Engineer, vol. 45, pp. 241-247.

Deryck, L. (1978). Natural propagation of electromagnetic waves in tunnels, IEEE Transactions on Vehicular Technology, vol. VT-27, pp. 145-150.

Damoso, E. \& Padova, S. (1976). Propagation and radiation of VHF radio signals in motorway tunnels, IEEE Trans. on Vehicular Technology, vol. VT-25, pp. 39-45. 
Mandich, D. \& Carpine, E. An experimental study of wave-guiding effect on radiowave propagation in arched railways tunnels at 450 and $900 \mathrm{MHz}$, Proceedings of the International Conference on Electromagnetic in Advanced Applications (ICEAA 95), pp. 69-72, Sept. 12-15, Politecnico di Torino, Italy, 1995.

Abo-Seida, O., Bishay, S. \& El-Morabie, K. (2006). IEEE Trans. Geosci. Remote Sensing 44 (8) pp. 2135-2142.

Abo-Seida, O. (2006). Journal of Electromagnetic Waves and Applications, 20 (6), pp.707-715.

Abo-Seida, O. (2002). Propagation of electromagnetic waves in a rectangular tunnel , ACES Journal, vol. 17, pp. 170-175.

Marcuvitz, N. (1951). Waveguide Handbook, Mc Graw-Hill, New York and London, pp. 5572. 


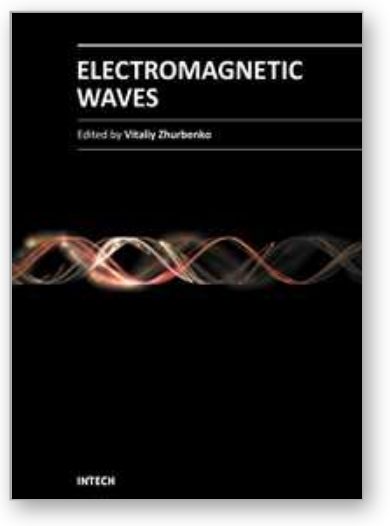

\author{
Electromagnetic Waves \\ Edited by Prof. Vitaliy Zhurbenko
}

ISBN 978-953-307-304-0

Hard cover, 510 pages

Publisher InTech

Published online 21, June, 2011

Published in print edition June, 2011

This book is dedicated to various aspects of electromagnetic wave theory and its applications in science and technology. The covered topics include the fundamental physics of electromagnetic waves, theory of electromagnetic wave propagation and scattering, methods of computational analysis, material characterization, electromagnetic properties of plasma, analysis and applications of periodic structures and waveguide components, and finally, the biological effects and medical applications of electromagnetic fields.

\title{
How to reference
}

In order to correctly reference this scholarly work, feel free to copy and paste the following:

Osama M. Abo-Seida (2011). Electromagnetic Wave Propagation in Circular Tunnels, Electromagnetic Waves, Prof. Vitaliy Zhurbenko (Ed.), ISBN: 978-953-307-304-0, InTech, Available from:

http://www.intechopen.com/books/electromagnetic-waves/electromagnetic-wave-propagation-in-circulartunnels

\section{INTECH}

open science | open minds

\section{InTech Europe}

University Campus STeP Ri

Slavka Krautzeka 83/A

51000 Rijeka, Croatia

Phone: +385 (51) 770447

Fax: +385 (51) 686166

www.intechopen.com

\section{InTech China}

Unit 405, Office Block, Hotel Equatorial Shanghai

No.65, Yan An Road (West), Shanghai, 200040, China

中国上海市延安西路65号上海国际贵都大饭店办公楼 405 单元

Phone: +86-21-62489820

Fax: $+86-21-62489821$ 
(C) 2011 The Author(s). Licensee IntechOpen. This chapter is distributed under the terms of the Creative Commons Attribution-NonCommercialShareAlike-3.0 License, which permits use, distribution and reproduction for non-commercial purposes, provided the original is properly cited and derivative works building on this content are distributed under the same license. 